\title{
Porous Channel Flows with Spontaneously Broken Symmetry
}

\author{
Eugen Magyari
}

Received: 21 April 2013 / Accepted: 3 September 2013 / Published online: 25 October 2013

C) Springer Science+Business Media Dordrecht 2013

\begin{abstract}
It is known that even entirely symmetric boundary value problems can admit solutions in which the inherent symmetry of the governing equations gets spontaneously broken. When this happens, two non-symmetric "twin solutions" occur so that the symmetry of the boundary value problem broken in the two individual solutions becomes restored by the twins. The present paper shows that, as a combined effect of buoyancy, viscous dissipation and pressure work, in a mixed convection flow in a vertical porous channel with isothermal walls, in addition to symmetric solutions, precisely this kind of twin solutions with broken symmetry can occur, although the walls are kept at the same temperature. The existence of another remarkable solution branch which describes symmetric adiabatic flows corresponding to nonlinear eigenvalues of the problem is also reported. The heat-work balance of all these steady-flow states is discussed in detail. The analysis is supported by an intuitive pointmechanical analogy. The equivalence of this mechanical energy analysis to the standard phase space method is also discussed shortly.
\end{abstract}

Keywords Mixed convection - Channel flow - Viscous dissipation · Pressure work · Spontaneous symmetry breaking $\cdot$ Adiabatic flow $\cdot$ Nonlinear eigenvalues

\section{Introduction and Problem Formulation}

When the governing equations and the boundary conditions of a channel flow problem are symmetric with respect to the reflection $y \rightarrow-y$ of the transverse coordinate $y$, not all the solutions must necessarily be symmetric with respect to the midplane $y=0$ of the channel. In addition to symmetric solutions, also such solutions can occur in which the inherent symmetry of the governing equations is broken. This happens "spontaneously", i.e. without any mathematical reason which would be obvious from the very beginning.

E. Magyari $(\bowtie)$

Departement Physik, Theoretische Physik, Universität Basel,

Klingelbergstrasse. 82, 4056 Basel, Switzerland

e-mail: magyari@bluewin.ch 
Fig. 1 Sketch of the vertical channel with symmetric isothermal boundary conditions

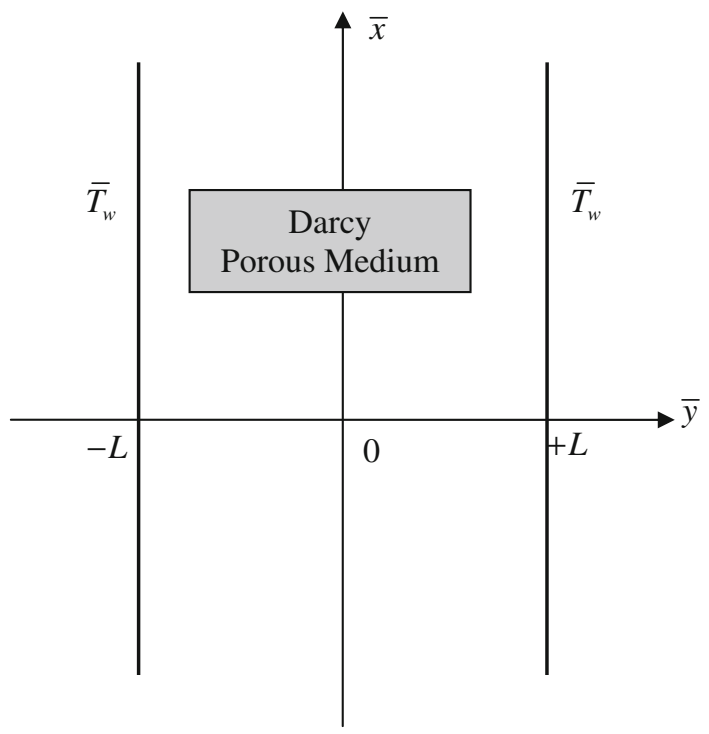

When, however, such a non-symmetric solution occurs, then also a second non-symmetric solution must exist so that these "twin solutions" restore the symmetry of the problem again. The present paper shows that in a mixed convection problem in a porous channel with isothermal walls, in addition to symmetric solutions precisely this kind of twin solutions with broken symmetry can occur, although the walls are kept at the same temperature. There also turns out that the physical reason for this spontaneous symmetry breaking resides in the possible occurrence of reverse-flow domains either in the left or the right half of the channel. However, in symmetric problems "left" and "right" being equivalent, the corresponding flow configurations with reverse-flow domains must occur pairwise so that the physical symmetry of the problem becomes restored again. The mathematical reason for this phenomenon is a bifurcation which results in the mentioned pair of asymmetric solutions.

To be specific we consider here the thermally fully developed mixed convection flow in a vertical parallel plane channel filled with a Darcy porous medium, assuming that the effect of viscous dissipation and pressure work are significant. This problem has recently been examined in a comprehensive paper of Barletta and Nield (2009a) assuming that the boundaries of the channel are subject to (non-symmetric) isothermal and isoflux boundary conditions, respectively. The exact solution of the problem has been given by Magyari (2009a) in terms of the Weierstrass elliptic P-function, and further special aspects have subsequently been discussed by Barletta and Nield (2009b) and by Magyari (2009b). The only change we introduce here in this model is the replacement of the mentioned non-symmetric boundary conditions by symmetric isothermal conditions, assuming that the walls of the channel (at $\bar{y}= \pm L$ ) are held at the same constant temperature $\bar{T}_{w}$. Our coordinate system and boundary conditions are schematically shown in Fig. 1. Thus, the governing Darcy-Boussinesq and energy equations of this boundary value problem for the flow velocity and temperature fields $\bar{u}=\bar{u}(\bar{y})$ and $\bar{T}=\bar{T}(\bar{y})$ still coincide with Eqs. (7) and (8) of Barletta and Nield (2009a),

$$
\frac{\mu}{K} \bar{u}=-\frac{\mathrm{d} \bar{p}}{\mathrm{~d} \bar{x}}-\rho\left[1-\beta\left(\bar{T}-\bar{T}_{w}\right)\right] g
$$


and

$$
k \frac{\mathrm{d}^{2} \bar{T}}{\mathrm{~d} \bar{y}^{2}}+\beta \bar{T} \bar{u} \frac{\mathrm{d} \bar{p}}{\mathrm{~d} \bar{x}}+\frac{\mu}{K} \bar{u}^{2}=0
$$

while the new boundary conditions read

$$
\bar{T}(-L)=\bar{T}(+L)=\bar{T}_{w}
$$

All the notations used in the above equations are standard and coincide with those of Barletta and Nield (2009a). The same holds for the dimensionless form of Eqs. (1)-(3),

$$
\begin{gathered}
u=1+R T \\
\frac{\mathrm{d}^{2} T}{\mathrm{~d} y^{2}}=B r(\varepsilon+R)(\gamma+T)(1+R T)-B r(1+R T)^{2} \\
T(-1)=T(+1)=0,
\end{gathered}
$$

where the dimensionless quantities involved have been defined as follows:

$$
\begin{aligned}
& y=\frac{\bar{y}}{L}, \quad u(y)=\frac{\bar{u}(\bar{y})}{\bar{u}_{0}}, \quad \bar{u}_{0}=-\frac{K}{\mu}\left(\frac{\mathrm{d} \bar{p}}{\mathrm{~d} \bar{x}}+\rho g\right), \quad T(y)=\frac{\bar{T}(\bar{y})-\bar{T}_{w}}{\Delta \bar{T}}, \\
& B r=\frac{\mu \bar{u}_{0}^{2} L^{2}}{k K \Delta \bar{T}}, \quad R=\frac{\rho g \beta K \Delta \bar{T}}{\mu \bar{u}_{0}}, \quad \gamma=\frac{\bar{T}_{w}}{\Delta \bar{T}}, \quad \varepsilon=\beta \Delta \bar{T}
\end{aligned}
$$

Here $\mathrm{d} \bar{p} / \mathrm{d} \bar{x}=$ const. is the applied pressure gradient, $B r$ is the Darcy-Brinkman number, $R$ the buoyancy ratio (of the Darcy-Rayleigh number to the Péclet number), $\Delta \bar{T}$ fixes the temperature scale of the problem while the parameters $\gamma$ and $\varepsilon$ compare this scale to the wall temperature $\bar{T}_{w}$ and to the thermal compressibility scale $\beta^{-1}$ of the fluid, respectively (see Barletta and Nield 2009a again). The first and second term on the right-hand side of Eq. (5) represents the contribution of the of the pressure work and the viscous dissipation, respectively. When both these effects are neglected, the unique solution of the problem is $T=0, u=1$, which means that in this case the fluid is in thermal equilibrium with the walls, $\bar{T}=\bar{T}_{w}$, and moves with uniform velocity $\bar{u}=\bar{u}_{0}$. It is also worth mentioning here that other porous channel flow models with a mathematical structure similar to that of the above Eq. (5) have also been investigated by Nield et al. (1996), Kuznetsov (1998), Umavathy et al. (2005, 2008) and Barletta et al. (2008).

According to Eqs. (4)-(6) the temperature problem is decoupled from the velocity problem so that the velocity field $u=u(y)$ is obtained in terms of the temperature solution $T=T(y)$ simply by Eq. (4). The Nusselt numbers $N u_{ \pm}=L \bar{q}( \pm L) /(k \Delta \bar{T})$ based on the half-width $L$ of the channel (where $\bar{q}(\bar{y})=-k \mathrm{~d} \bar{T} / \mathrm{d} \bar{y}$ stands for the dimensional heat flux in the transverse direction) are given in terms of the dimensionless temperature solution as

$$
N u_{ \pm}=-T^{\prime}( \pm 1)
$$

(the prime denotes differentiation with respect to $y$ ).

Furthermore, it is also clearly seen that the governing Eqs. (5) and (6) are invariant with respect to the reflection $y \rightarrow-y$ of the transverse coordinate $y$. Therefore, when $T(y)$ is a solution, then $T(-y)$ is also a solution of the temperature problem $(5,6)$. However, there is no general guarantee that in a symmetric boundary value problem the two solutions $T(y)$ and $T(-y)$ are always identical and, if they are, there is no guarantee that the corresponding symmetric solution $T(y)=T(-y)$ is the unique solution of the problem. In other words, nothing ensures from the very beginning that a symmetric boundary value problem admits only solutions which are symmetric with respect to the midplane $y=0$ of the coordinate system (more 
precisely, solutions which are even functions of $y$ ). It can happen that in the individual solutions $T(y)$ and $T(-y)$ the inherent symmetry of the problem is spontaneously broken, i.e., except for the values at the boundaries $y= \pm 1$ and at some isolated points, within the channel, the property $T(y) \neq T(-y)$ holds. When this really happens, $T(y)$ and $T(-y)$ represent precisely the asymmetric solution pair which restore the physical symmetry of the problem. One of the objectives of the present paper is a detailed analysis of this issue. The approach will be supported by an intuitive point-mechanical analogy. The equivalence of this mechanical energy analysis to the standard phase space method will be discussed in the Appendix.

\section{Prospecting the Solution Domain Guided by a Mechanical Analogy}

In order to investigate the structure of the solution space with the aim to predict the possible solution branches, it is convenient to transcribe Eq. (5) in the form

$$
\frac{\mathrm{d}^{2} T}{\mathrm{~d} y^{2}}=A_{3} T^{2}+A_{2} T-A_{1} \equiv-\frac{\partial W}{\partial T},
$$

where

$$
W(T)=-\frac{A_{3}}{3} T^{3}-\frac{A_{2}}{2} T^{2}+A_{1} T
$$

and

$$
A_{3}=\varepsilon R B r, \quad A_{2}=[\varepsilon-R+\gamma R(\varepsilon+R)] B r, \quad A_{1}=[1-\gamma(\varepsilon+R)] B r
$$

Now we can reinterpret the boundary value problem $(5,6)$ in an intuitive way as the pointmechanical problem of a one-dimensional motion. In this sense Eq. (9) describes the conservative motion of a particle of mass 1 , space coordinate $T$, and potential energy $W$. The transverse coordinate $y$ of the channel plays in this analogy the role of time variable of the particle motion and thus only the finite time interval $-1 \leq y \leq 1$ is of physical interest. In this context it is immediately clear that Eq. (9) admits a first integral which is the energy integral of the particle motion

$$
\frac{1}{2}\left(\frac{\mathrm{d} T}{\mathrm{~d} y}\right)^{2}+W(T)=E
$$

The constant of integration $E$ represents the conserved energy of the particle. Bearing in mind the boundary conditions (6) we have

$$
\left.W\right|_{y=-1}=\left.W\right|_{y=+1}=0
$$

Thus, Eqs. (12) and (8) imply the following relationship between the total energy $E$, the initial and final kinetic energies of the particle, as well as the Nusselt numbers at the boundaries of the channel:

$$
E=\frac{1}{2}\left[T^{\prime}(-1)\right]^{2}=\frac{1}{2} N u_{-}^{2}=\frac{1}{2}\left[T^{\prime}(+1)\right]^{2}=\frac{1}{2} N u_{+}^{2}
$$

All the possible solution branches of the problem $(1,2)$ can be predicted qualitatively by a simple inspection of Fig. 2 where the potential energy $W$ of the particle has been plotted as a function of its "space coordinate" $T$ for some positive values of the parameters $A_{1}, A_{2}$ and $A_{3}$ (which is the relevant range for the present study).

According to the boundary conditions (6) and Eqs. (13) the particle motion begins at the initial instant $y=-1$ in the origin $(T, W)=(0,0)$ and at the final instant $y=1$ the particle 


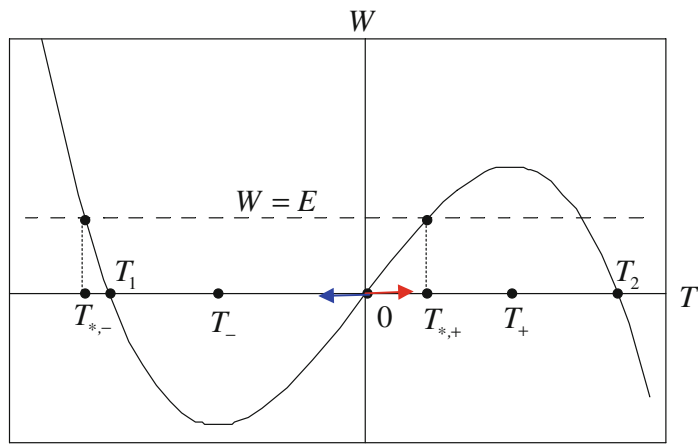

Fig. 2 Schematic plot of the particle potential energy (10) as a function of the particle "coordinate" $T$. Here $T_{*,+}$ and $T_{*,-}$ denote the right and left particle turning points which are solutions of the equation $W(T)=$ $E$. Furthermore, $T_{ \pm}=-\left(A_{2} / 2 A_{3}\right) \pm \sqrt{\left(A_{2} / 2 A_{3}\right)^{2}+\left(A_{1} / A_{3}\right)}$ are the coordinates of the maximum and minimum of $W$, and $T_{1,2}=-\left(3 A_{2} / 4 A_{3}\right) \mp \sqrt{\left(3 A_{2} / 4 A_{3}\right)^{2}+\left(3 A_{1} / A_{3}\right)}$ and $T_{3}=0$ are the zeros of $W(T)$

has to reach (or to pass) the origin again. In the context of our boundary value problem, in the range $y>1$ the particle motion is meaningless. Obviously, the particle can be started either to the right or to the left with some (positive or negative) initial velocity $T^{\prime}(-1)$. Then, at $y=1$ the particle has to reach the origin again with some end velocity $T^{\prime}(+1)$. These (yet unknown) velocities are related to the respective Nusselt numbers by Eqs. (8). A systematic discussion of the possible particle motions is the objective of the next Sections. The main focus is the identification of the symmetry breaking solutions in addition to the symmetric solution branches.

\section{Particle Motions Associated with Symmetric Solutions}

In case $T^{\prime}(-1)>0$, the particle starts to move to the right (red arrow of Fig. 2), reaches at some instant $y=y_{*,+}$ a turning point at a distance $T=T_{*,+}>0$ from the origin where its velocity vanishes, $T^{\prime}\left(y_{*,+}\right)=0$, and then changes sign, moving toward the origin again. When the origin is passed precisely at $y=1$, the particle motion specifies a solution of the flow and heat transfer problem. In this case $T$ is positive for all $-1<y<1$ and $T^{\prime}(+1)=-T^{\prime}(-1)<0$ which means that the temperature profile is symmetric and nodeless. Accordingly, $y_{*,+}=0$, and $T_{*,+}$ is precisely the largest temperature of the fluid, $T_{*,+}=T_{\max }$. Equation (14) applied for $y=y_{*,+}$ gives

$$
W\left(T_{\max }\right)=E=\frac{1}{2}\left[T^{\prime}(-1)\right]^{2}=\frac{1}{2}\left[T^{\prime}(+1)\right]
$$

The corresponding symmetric solution is obtained from Eqs. (12) and (15) in the implicit form

$$
y=\mp\left(1-\frac{1}{\sqrt{2}} \int_{0}^{T} \frac{\mathrm{d} T}{\sqrt{W\left(T_{\max }\right)-W(T)}}\right)
$$

where the signs $\mp$ correspond to the ranges $-1 \leq y \leq 0$ and $0 \leq y \leq 1$, respectively.

Now applying Eq. (16) for $y=y_{*,+}=0$, we obtain for determination of the maximum temperature the equation 


$$
\frac{1}{\sqrt{2}} \int_{0}^{T_{\max }} \frac{\mathrm{d} T}{\sqrt{W\left(T_{\max }\right)-W(T)}}=1
$$

The corresponding initial particle velocity $T^{\prime}(-1)$ results then from Eq. (15) as

$$
T^{\prime}(-1)=\sqrt{2 W\left(T_{\max }\right)}
$$

Because of its analogy to the well-known relationship which relates the initial particle velocity to the maximum height reached by a particle in the vertical throw, Eq. (18) will be named hereafter as Galilei formula. Equations (16)-(18) describe the simplest solution branch of the problem completely. This will be referred to as the first symmetric solution branch. It is also worth noticing here that the largest temperature of the flow $T_{\max }=T_{*,+}$ never can reach or exceed the value $T=T_{+}$corresponding to the maximum of the particle potential energy. Indeed, would the particle pass the point $T=T_{+}$, it would depart inevitably to infinity. Would the particle reach the point $T=T_{+}$with zero velocity, it would remain at rest at this equilibrium point forever. Therefore, we have necessarily $T_{\max } \equiv T_{*,+}<T_{+}$for all the possible particle motions.

Now let us assume that the particle motion is still started to the right but with an initial velocity $T^{\prime}(-1)$ which is a little bit smaller than that needed for the first branch solution described above. In this case the particle returns to the origin $(T, W)=(0,0)$ earlier than at $y=1$ (where the first node of the function $T(y)$ occurs). Then the motion continues to the left until at some instant $y=y_{*,-}$ a second particle turning point $T_{*,-}$ is reached (see Fig. 2). When there happens that $y_{*,-}=0$, we obtain a solution of the second symmetric branch. This left particle turning point corresponds to the smallest temperature of the fluid, $T_{*,-}=T_{\min }$ which similarly to $T_{*,+}=T_{\max }$, is a root of equation (15). Thus we always have $T_{*,-}=T_{\min }<T_{1}$ except for $E=0$ where $T_{\min }=T_{1}$ holds (see also Sect. 5). Subsequently the particle moves to the right, passes the origin with the velocity $T^{\prime}(-1)$ (where a second node of the function $T\left(y\right.$ )occurs), reaches the first turning point $T_{*,+}=T_{\max }$ again, and returns to the origin precisely at the instant $y=1$ with the velocity $T^{\prime}(1)=$ $-T^{\prime}(-1)$. The red and green temperature profiles seen in Fig. 3 are symmetric first and second branch solutions, respectively, and illustrate the above two mechanical motions for the set of the parameter values $\left(A_{1}, A_{2}, A_{3}\right)=(1,1,1000)$. The corresponding initial velocities are $T^{\prime}(-1)=0.20292684$ and $T^{\prime}(-1)=0.19977756$, respectively. The maxima and minima of the temperature profiles correspond to the passage times $y_{*}$ of the particle through the turning points, while the nodes of $T(y)$ correspond to the passages through the origin.

Now, after these explanations, it is easy to imagine what happens when the particle motion is started again to the right but with a certain initial velocity which is smaller that the latter one, $T^{\prime}(-1)=0.19977756$. Well, by doing this with $T^{\prime}(-1)=0.09758345$, the particle reaches the final state $T(+1)=0$ after one loop more and we obtain the temperature profile given by the blue curve of Fig. 3. This is an example for a symmetric third branch solution which is described by a function $T(y)$ having four nodes. While the first branch (nodeless) solution describes a unidirectional "direct flow", the second and third branch solutions having, respectively, two and four nodes correspond to bidirectional flows with one and two reverse-flow domains between the nodes. Our mechanical analogy also suggests that depending on the choice of the parameters $\left(A_{1}, A_{2}, A_{3}\right)$ the sequence of the symmetric solutions $T=T(y)=T(-y)$ with an increasing number of nodes can be continued indefinitely. Although we speak about "flow domains", in Fig. 3 we see only "temperature domains" of the flow. However, these low- and high-temperature domains can easily be converted in velocity domains of the flow with the aid of Eq. (4), $u=1+R T$. 


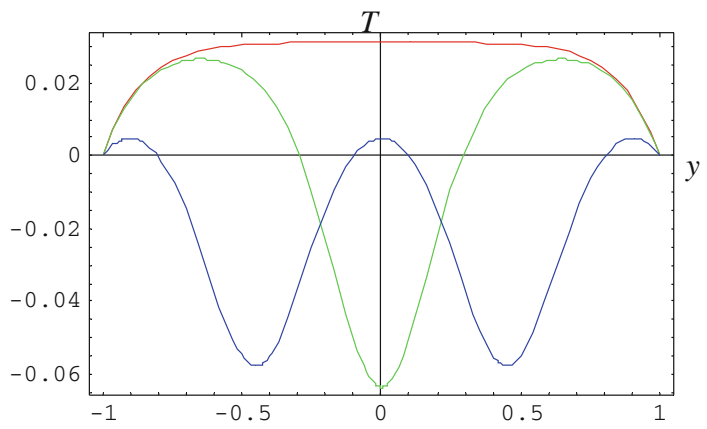

Fig. 3 Symmetric temperature solutions of the first branch (red curve, nodeless), second branch (green curve, two nodes) and third branch (blue curve, four nodes), all associated with the same parameter values $\left(A_{1}, A_{2}, A_{3}\right)=(1,1,1000)$. The corresponding initial velocities (wall heat fluxes at $\left.y=-1\right)$ are $T^{\prime}(-1)=$ $0.20292684, T^{\prime}(-1)=0.19977756$ and $T^{\prime}(-1)=0.09758345$, respectively

Before turning to the symmetry breaking solutions it is worth describing here the special case of the forced convection corresponding to $R=0$. In this case $A_{3}=0$ and the boundary value problem $(5,6)$ becomes linear in $T$. The particle potential energy (10) reduces to $W(T)=-\left(A_{2} / 2\right) T^{2}+A_{1} T$ which is a parabola having at $T_{+}=A_{1} / A_{2}$ a maximum $W\left(T_{+}\right)=W_{\max }=A_{1}^{2} /\left(2 A_{2}\right)$. Compared with the nonlinear mixed convection case $\left(A_{3} \neq\right.$ 0 ) the significant change of the particle potential energy curve of Fig. 2 consists now of the fact that the left minimum of $W$ disappears and the whole left branch of $W$ is stretched to the left branch of the parabola approaching $-\infty$ as $T \rightarrow-\infty$. As a consequence, the motion can only be started with a positive initial particle velocity $T^{\prime}(-1)>0$ (otherwise, the particle can never return to the origin, i.e., the boundary condition $T(+1)=0$ cannot be satisfied for $\left.T^{\prime}(-1) \leq 0\right)$. The corresponding temperature solution $T$ is positive for all $-1<y<1$, with $T^{\prime}(-1)=-T^{\prime}(1)>0$, it is unique and belongs to the first symmetric solution branch. The integrals occurring in Eqs. (16) and (17) can be expressed in this case by elementary functions. Thus, Eq. (17) yields for the maximum temperature $T_{\max }=T_{*}$ (particle turning point) the result

$$
T_{\max }=\frac{A_{1}}{A_{2}}\left[1-\frac{1}{\cosh \left(\sqrt{A_{2}}\right)}\right]
$$

which explicitly shows that $T_{\max }<T_{+}=A_{1} / A_{2}$. The corresponding initial particle velocity is obtained from the Galilei formula (18) as

$$
T^{\prime}(-1)=\frac{A_{1}}{\sqrt{A_{2}}} \tanh \left(\sqrt{A_{2}}\right)
$$

The forced convection temperature field results after elementary operations from Eq. (16) in the form

$$
T(y)=\frac{A_{1}}{A_{2}}\left[1-\frac{\cosh \left(\sqrt{A_{2}} y\right)}{\cosh \left(\sqrt{A_{2}}\right)}\right]=\left(\frac{1}{\varepsilon}-\gamma\right)\left[1-\frac{\cosh (\sqrt{\varepsilon B r} y)}{\cosh (\sqrt{\varepsilon B r})}\right]
$$

It is clearly seen that Eq. (21) specifies an even function of the transverse coordinate $y$ (similarly to the red curve of Fig. 2). At the same time one realizes that all the interesting physical and mathematical features of the boundary value problem $(5,6)$ can only occur in 
the nonlinear mixed convection case $A_{3} \neq 0$. Because it is proportional to the buoyancy ratio $R$, the key parameter $A_{3}$ will be referred hereafter to as the buoyancy parameter.

\section{Solutions with Spontaneously Broken Symmetry}

The description of the particle motions corresponding to the solutions $T=T(y)$ seen in Fig. 3 raises the following question: what happens when the motion is started to the right with a new initial particle velocity $T^{\prime}(-1)$ which is somewhere between the initial velocities of the symmetric first and second branch solutions, i.e., $0.19977756<T^{\prime}(-1)<0.20292684$ ? Based on the Figs. 2 and 3 our expectation is that, for a suitable value of $T^{\prime}(-1)$ and after passing the minimum of the potential energy, the particle could reach the origin at the final instant $y=1$, i.e., without to cover the distance from 0 to the right turning point and back to the origin again (as it happens for the symmetric second branch solutions). Such a motion would correspond to a "stretching" of the green curve of Fig. 3 so that its right maximum and the right node (and thus its symmetry) are removed and the point $T(+1)=0$ is approached from below. This is precisely what happens. Staring the motion with particle velocity $T^{\prime}(-1)=+0.20291585419$ we obtain the non-symmetric solution given by the red curve of Fig. 4. Now it is clear that by starting the motion with the opposite initial particle velocity, $T^{\prime}(-1)=-0.20291585419$, one obtains the blue curve of Fig. 4 . These non-symmetric "twins" of Fig. 4 with each one node restore the symmetry of the problem which has been broken by the individual solutions.

Because in the above example the initial particle velocities of the non-symmetric solutions and those corresponding to the first and second symmetric branches are very close to each other, the numerical calculations have to be performed with an enhanced precision. However, the same calculations become less sensitive when instead of $\left(A_{1}, A_{2}, A_{3}\right)=(1,1,1000)$ the parameter values $\left(A_{1}, A_{2}, A_{3}\right)=(1,1,100)$ are used. The corresponding results showing the first and second symmetric branch solutions as well as the non-symmetric twins in between have been plotted in Fig. 5 .

\section{Nonlinear Eigenvalues and Adiabatic Flows with $T(y) \leq 0$}

When the motion is started to the left (the blue arrow of Fig. 2) with a non-vanishing initial particle velocity $T^{\prime}(-1)<0$, it is obvious that in a suitable range of the governing parameter solutions must exist which describe symmetric flows with reverse velocity domains for which

Fig. 4 Twin solutions with broken symmetry corresponding to the parameter values $\left(A_{1}, A_{2}, A_{3}\right)=(1,1,1000)$ and initial velocities

$T^{\prime}(-1)=+0.20291585419$ (red curve $T(y))$ and

$T^{\prime}(-1)=-0.20291585419$

(blue curve $T(-y)$ ), respectively

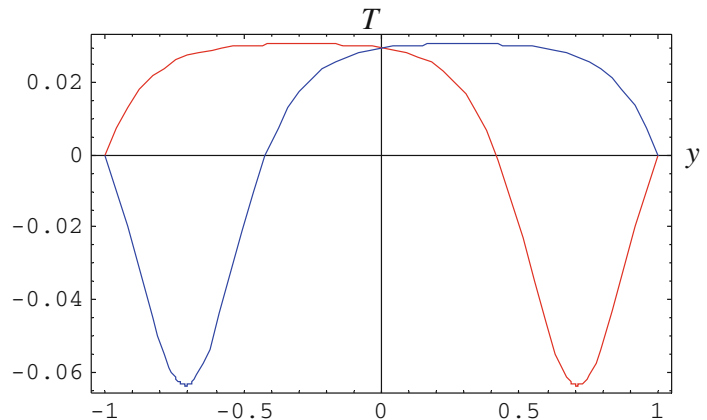




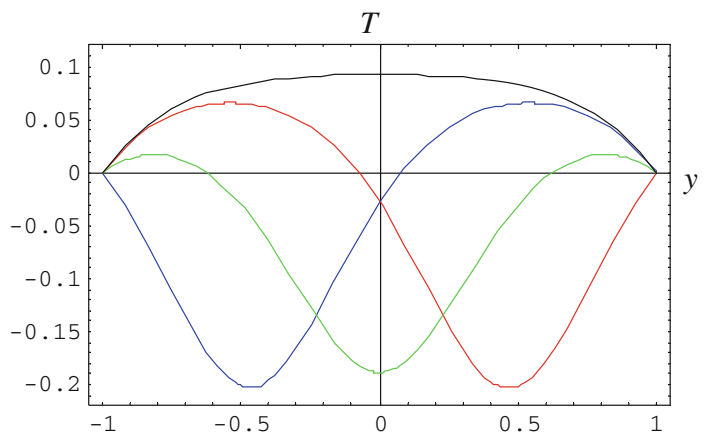

Fig. 5 Four temperature solutions corresponding to the parameter values $\left(A_{1}, A_{2}, A_{3}\right)=(1,1,100)$. The black and green curves are symmetric first and second branch solutions and correspond to the initial velocities $T^{\prime}(-1)=0.351685$ and $T^{\prime}(-1)=0.183253$ of the equivalent particle motions, respectively. The twin solutions with spontaneously broken symmetry correspond to $T^{\prime}(-1)=+0.329160$ (red curve) and $T^{\prime}(-1)=-0.329160$ (blue curve), respectively

Fig. 6 Symmetric nodeless solutions $T(y)<0$ for $A_{1}=A_{2}=1$ and the indicated values of the buoyancy parameter $A_{3}$. The corresponding initial velocities are

$T^{\prime}(-1)=-0.553207$ (red curve) and $T^{\prime}(-1)=-0.200923($ green curve), and $T^{\prime}(-1)=-0.063143$ (blue curve)

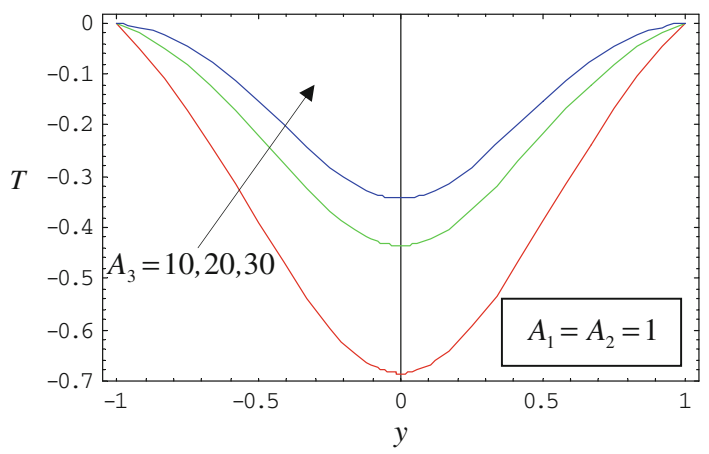

$T(y) \leq 0$ for all $y \leq 1$ holds. The particle energy conservation (14) implies in this case $T^{\prime}(+1)=-T^{\prime}(-1)>0$. Some examples illustrating such flow regimes are shown in Fig. 6 . For $A_{1}=A_{2}=1$, both $T_{1}$ and the minimum $\left|W\left(T_{-}\right)\right|$of the particle potential energy $W$ decrease with increasing values of the buoyancy parameter $A_{3}$. Therefore, the larger the $A_{3}$ the smaller the initial particle velocity $\left|T^{\prime}(-1)\right|$. Otherwise, the particle returns to the origin earlier than at the instant $y=1$. This explains the features of the curves plotted in Fig. 6.

The behavior seen in Fig. 6 also suggests that (for $A_{1}=A_{2}=1$ ) in the range $A_{3}>30$ a well-defined value $A_{3}^{(1)}$ of the buoyancy parameter $A_{3}$ may exist so that such a symmetric nodeless solution becomes possible only for a vanishing initial particle velocity $T^{\prime}(-1)=0$, i.e., for $E=0$. This means that the particle turning point $T_{*,-}=T_{\min }$ coincides with the left zero $T_{1}$ of the particle potential energy $W, T_{*,-}=T_{\min }=T_{1}$, (see Fig. 2) and this is reached at the instant $y_{*,-}=0$. The particle energy conservation requires in this case $T^{\prime}(+1)=T^{\prime}(-1)=0$ which (according to Eqs. 8) means that no heat is transferred through the walls of the channel, i.e., the flow is adiabatic. The question is how the value $A_{3}^{(1)}$ associated with this adiabatic flow can be determined.

From mechanical point of view the particle motion started at $T=0$ with vanishing initial velocity (and thus with $E=0$ ) is a nonlinear oscillation between the points of coordinates $T=0$ and $T=T_{1}$. For specified values of the parameters $A_{i}, i=1,2,3$, the period $P$ of these oscillations can be determined by integrating Eq. (12) with respect to $y$ from the initial 
instant $y=-1$ corresponding to the initial position $T=0$ to $y=y_{*,-}=0$ corresponding to the particle turning point $T_{*,-}=T_{1}$. Thus we obtain for the half period

$$
\frac{P}{2}=-\frac{1}{\sqrt{2}} \int_{0}^{T_{1}} \frac{\mathrm{d} T}{\sqrt{-W(T)}}
$$

By the substitution $T=T_{1} \sin ^{2} \theta$, Eq. (22) becomes

$$
\frac{P}{2}=\sqrt{\frac{6}{T_{2} A_{3}}} \int_{0}^{\pi / 2} \frac{\mathrm{d} \theta}{\sqrt{1-\left(T_{1} / T_{2}\right) \sin ^{2} \theta}},
$$

where $T_{2}$ is the largest positive root of equation $W(T)=0$ (see the caption of Fig. 2). The integral occurring in Eq. (23) is the complete elliptic integral of the first kind $F\left(\pi / 2 \mid T_{1} / T_{2}\right)$ (see Abramowitz and Stegun 1972, Eqs. 17.3.1 and 17.3.2) and thus

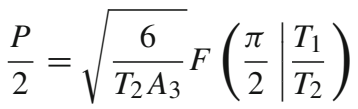

Now the special value $A_{3}^{(1)}$ of the buoyancy parameter corresponding to the above-described symmetric nodeless solution is obtained from Eq. (22) by requiring that, according to the boundary conditions (6), the period equals exactly two time units, i.e., $P=2$. The solution of Eq. (24) in this case (for $A_{1}=A_{2}=1$ ) is $A_{3} \equiv A_{3}^{(1)}=37.6952$. The temperature profile of the adiabatic flow corresponding to the value $A_{3}^{(1)}$ of the buoyancy parameter is represented by the black curve of Fig. 7. The corresponding exact solution in implicit form reads

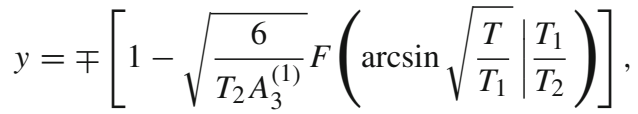

where the signs $\mp$ correspond to the ranges $-1 \leq y \leq 0$ and $0 \leq y \leq 1$, respectively, and $F(\varphi \mid m)$ stands for the elliptic integral of the first kind (see Abramowitz and Stegun 1972, Eqs. 17.2.6 and 17.2.1).

As stated in Section 2.4, both $T_{1}$ and the minimum $\left|W\left(T_{-}\right)\right|$of the particle potential energy $W$ decrease with increasing values of the buoyancy parameter $A_{3}$. Therefore, when $A_{3}>A_{3}^{(1)}$, the period $P$ of the nonlinear oscillation becomes smaller than 2 and thus the boundary condition $T(+1)=0$ cannot be satisfied at the end of the first period. Accordingly, in the range $A_{3}>A_{3}^{(1)}$ there can occur further adiabatic temperature solutions only when the final instant of motion $y=1$ is reached after 2,3, or in general after an integer number $n$ of oscillation periods. In this way one obtains in addition to $A_{3}^{(1)}$ further nonlinear eigenvalues $A_{3}^{(n)}$ which can be calculated from Eq. (24) by replacing $P$ by $P_{n}=2 / n$, i.e., from equation

$$
\sqrt{\frac{6}{T_{2} A_{3}^{(n)}}} F\left(\frac{\pi}{2} \mid \frac{T_{1}}{T_{2}}\right)=\frac{1}{n}, \quad n=1,2,3, \ldots
$$

Setting here $n=2,3$ and 4, one obtains the eigenvalues $A_{3}^{(2)}=576.5296, A_{3}^{(3)}=2892.6345$, and $A_{3}^{(4)}=9113.0696$. The corresponding adiabatic temperature profiles are given by the green, blue, and the red curve of Fig. 7, respectively. The $n$th temperature profile has $n-1$ maxima, at each of which $T(y)=0$ and $T^{\prime}(y)=0$. These maxima correspond to the 
Fig. 7 Adiabatic temperature profiles for $A_{1}=A_{2}=1$ and the nonlinear eigenvalues of the buoyancy parameter $A_{3}$ :

$A_{3}^{(1)}=37.6952$ (black curve, no intermediary maxima),

$A_{3}^{(2)}=576.5296$ (green curve,

one maximum)

$A_{3}^{(3)}=2892.6345$ (blue curve,

two maxima) and

$A_{3}^{(4)}=9113.0696$ (red curve, three maxima)

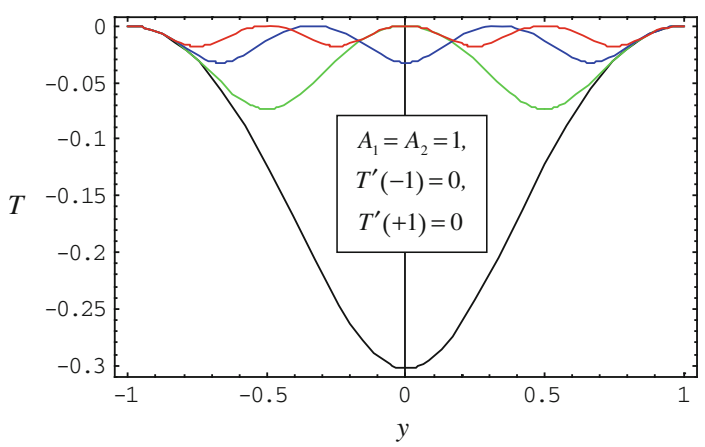

instants at which the particle is at rest at the origin. The eigenvalues $A_{3}^{(n)}$ of the buoyancy parameter correspond (in the mechanical analogy) to the oscillation frequencies $1 / P_{n}=$ $n / 2=1 / 2,1,3 / 2,2, \ldots$ of the particle.

\section{The Heat-Work Balance of the Temperature Solutions}

The mixed convection flow is driven by the applied pressure gradient and the thermal buoyancy. In the present case the temperature gradient which sustains the buoyancy force results as a combined effect of the heat supply/removal through the boundaries, of the volumetric heat generation by viscous dissipation (frictional heat) and the heat released/consumed by the pressure work. The goal of this Section was to discuss the temperature solutions reported above with regard to this complex interplay between the heat supplied through the walls on the one hand and the effect of viscous dissipation and pressure work on the other hand.

Integrating the energy balance equation (5) with respect to $y$ from -1 to 1 and bearing in mind Eq. (8) one easily obtains

$$
N u_{+}-N u_{-}=q_{\text {diss }}+q_{\text {work }}
$$

The left-hand side of this equation represents the net (dimensionless) heat flux passing the walls of the channel, and the right-hand side of Eq. (27) where

$$
\begin{gathered}
q_{\mathrm{diss}}=B r \int_{-1}^{1}(1+R T)^{2} \mathrm{~d} y, \\
q_{\text {work }}=-B r \int_{-1}^{1}(\varepsilon+R)(\gamma+T)(1+R T) \mathrm{d} y
\end{gathered}
$$

specifies the contributions of viscous dissipation and pressure work to the net heat flux transferred through the channel. Obviously, $q_{\text {diss }}$ is always positive, while $q_{\text {work }}$ can either be positive or negative. Furthermore, according to Eq. (14) the two Nusselt numbers can differ from each other at most in their sign,

$$
\left|N u_{+}\right|=\left|N u_{-}\right|
$$

Now, with the aid of Eqs. (27)-(30), the detailed heat-work balance can be discussed for all the solutions reported in the paper easily. For the already specified values of $A_{1}, A_{2}$, and 
$A_{3}$, the values of the physical parameters $\varepsilon, \gamma$ and $R$ can be calculated as functions of the Darcy-Brinkman number $\mathrm{Br}$ from the system of the definition equations (11).

In case of all the symmetric solutions plotted in Figs. 3,4 and 5 one has $N u_{+}=-N u_{-}=$ $T^{\prime}(-1)>0$, i.e., outward heat fluxes through both the boundaries of the channel. This implies in turn $q_{\text {diss }}+q_{\text {work }}=-2 N u_{-}=2 T^{\prime}(-1)>0$. For example, in case of the black temperature profile of Fig. 5 we have $2 T^{\prime}(-1)=0.703372$. Bearing in mind that in this case $\left(A_{1}, A_{2}, A_{3}\right)=(1,1,100)$, Eq. (11) yield for the parameters (for $B r=5$, e.g.) the values $\varepsilon=2.1925, \gamma=0.0688765$, and $R=9.51249$. Thus we obtain for the contribution of the viscous dissipation and the pressure work to the net wall heat flux $2 T^{\prime}(-1)=0.70337$ the values $q_{\text {diss }}=28.884451$ and $q_{\text {work }}=-28.181081$. Similarly, for the symmetric temperature profile represented by the green curve of Fig. 5 we obtain $2 T^{\prime}(-1)=0.36651, q_{\text {diss }}=$ $6.87828, q_{\text {work }}=-6.51177$. In both of these cases the frictional heat generation exceeds (moderately) the heat consumed by the expansion work. The excess heat flows outward through the isothermal walls of the channel. Therefore, in these symmetric flow states the channel works as a heating device (heat release through the boundaries).

As expected, for the symmetric reverse flow states plotted in Fig. 6, the opposite is true, i.e., the channel works as a cooling device. Indeed, in these cases we have $N u_{+}=-N u_{-}=$ $T^{\prime}(-1)<0$, i.e., inward heat fluxes through both the boundaries of the channel. This implies in turn $q_{\text {diss }}+q_{\text {work }}=-2 N u_{-}=2 T^{\prime}(-1)<0$. For example, in case of the red temperature profile of Fig. 6 we have $2 T^{\prime}(-1)=-1.106414$. Bearing in mind that in this case $\left(A_{1}, A_{2}, A_{3}\right)=(1,1,10)$, Eq. (11) yield for the parameters (for the same $\left.B r=5\right)$ the values $\varepsilon=0.740312, \gamma=0.232431$ and $R=2.70156$. Thus we obtain for the contribution of the viscous dissipation and the pressure work to the net heat flux $2 T^{\prime}(-1)=-1.1064$ the values $q_{\text {diss }}=3.67936$ and $q_{\text {work }}=-4.7858$ which shows that the heat consumed by the expansion work exceeds the frictional heat. In this case, the excess work is done by the inward heat fluxes through the isothermal walls. Thus, in the symmetric reverse-flow states of Fig. 6 the channel works indeed as a cooling device (heat absorption across the boundaries).

Let us now consider the asymmetric pair of solutions represented by the red and blue curves of Fig. 5. For example, in case of the blue temperature profile we have $\mathrm{Nu} u_{+}=$ $N u_{-}=-T^{\prime}(-1)>0$, i.e., an inward heat flux through the left and an outward one through the right boundary of the channel. This implies in turn $q_{\text {diss }}+q_{\text {work }}=0$ which means that the heat generated by viscous friction is compensated exactly by the heat consumed by the expansion work. For the same value $\mathrm{Br}=5$ of the Brinkman number we obtain in this case $q_{\text {diss }}=-q_{\text {work }}=11.804$. Therefore, in the asymmetric states the channel works as an ideal heat transfer device $\left(N u_{+}=N u_{-}>0\right.$, blue curve, heat transfer from left to right; $N u_{+}=N u_{-}<0$, red curve, heat transfer from right to left). At the first sight, this result seems to be physically contradictory since the boundaries are isothermal and symmetric, the frictional heat is consumed by the expansion work and, in spite of this fact, we have a non-vanishing heat flux through the channel, $N u_{+}=N u_{-} \neq 0$. The explanation, however, is simple. The mutually compensating fluxes $q_{\text {diss }}$ and $q_{\text {work }}$ given by Eqs. (28) and (29) are integral quantities. Their local counterparts, however, are functions of non-uniform temperature distributions $T(y)$ (the red or blue curves of Fig. 6) and thus variable quantities. The non-vanishing heat transfer through the channel originates from this local non-uniformity.

The fourth type of solutions, the adiabatic temperature profiles illustrated in Fig. 7, correspond to the converse case of the asymmetric solutions in the sense that now $\mathrm{Nu}_{+}=$ $N u_{-} \equiv 0$, and thus still $q_{\text {diss }}+q_{\text {work }}=0$ holds. This means that the channel works in this case as an ideal insulator, in contrast to the ideal heat transfer device of the case $\left(q_{\text {diss }}+q_{\text {work }}=0, N u_{+}=N u_{-} \neq 0\right)$ corresponding to the asymmetric temperature distributions. For example, in case of the black curve of Fig. 7 corresponding to $A_{3}=37.6952$ 
Eq. (11) yield (for the same $B r=5$ ) the parameters the values $\varepsilon=1.332, \gamma=0.114417$, and $R=5.65998$. The corresponding mutually compensating integral fluxes are $q_{\text {diss }}=$ $-q_{\text {work }}=4.1578$ while at the boundaries $T^{\prime}( \pm 1)=0$ holds.

\section{Summary and Conclusions}

In the present paper, the fully developed mixed convection flow in a vertical parallel plane channel filled with a Darcy porous medium has been considered assuming that (i) the walls of the channel are held at the same constant temperature and (ii) the effects of viscous dissipation and pressure work are significant. The investigation of the solution domain of this nonlinear flow and temperature problem was guided by an intuitive point mechanical analogy (see Sect. 2) which is the "physicist's version" of the standard mathematical method of the "phase portrait analysis" (see Appendix). The main results of the paper can be summarized as follows:

1. In a physically relevant range of the governing parameters a sequence of solution branches have been found which are symmetric with respect to the midplane $y=0$ of the channel (see Figs. 3, 5, 6, 7).

2. Although the governing differential equation as well as the boundary conditions is symmetric with respect to the reflection $y \rightarrow-y$ of the transverse coordinate $y$, in addition to the mentioned symmetric solutions also pairs of asymmetric solutions can occur in which the basic left-right symmetry of the problem gets spontaneously broken (see the red and blue curves of Figs. 4, 5).

3. The reduced Nusselt number $N u=\left|T^{\prime}(-1)\right|$ corresponding to the asymmetric solutions is located between the Nuseelt numbers $\left|T^{\prime}(-1)\right|$ corresponding to two subsequent symmetric solution branches (see, e.g. Fig. 5).

4. The physical reason for the symmetry breaking resides in the possible occurrence of reverse flow regions either in the left or the right half of the flow domain. However, in symmetric problems "left" and "right" being equivalent, the corresponding flow configurations with reverse flow must occur pairwise so that the physical symmetry of the problem becomes restored again (see Figs. 4, 5).

5. For a denumerable set of values $A_{3}^{(n)}, n=1,2,3, \ldots$ of the buoyancy parameter, referred to as nonlinear eigenvalues of the problem, symmetric solutions were found which describe adiabatic flows (see Fig. 7). In terms of the mechanical analogy the adiabatic temperature solutions describes nonlinear oscillations of the particle with frequencies $n / 2=1 / 2,1,3 / 2,2, \ldots$.

6. The integral heat-work balance of the steady temperature solution branches identified in the paper reveals that the channel works as a heating device in the symmetric flow states, as a cooling device in the reverse flow states, as an ideal heat transfer device in the asymmetric flow states, and as an ideal insulator in the adiabatic flow states. In the first case the frictional heat generation exceeds the heat consumed by the expansion work, in the second case the opposite holds, while in the latter two cases the heat generated by viscous friction is compensated exactly by the heat consumed by the expansion work.

\section{Appendix: Prospecting the Solution Domain by the Phase Portrait Analysis}

The phase portrait analysis is the standard method for the qualitative study of autonomous second-order ordinary differential equations o the form $\ddot{x}=f(x, \dot{x})$. It enables one to obtain 
the basic characteristics of the solutions without actually solving the differential equation. The geometrical device of the phase portrait analysis is the phase plane $(x, \dot{x})$. The phase paths $\dot{x}=$ functionof $(x)$ (called also trajectories, orbits, integral curves) reveal the basic properties of the solutions of equation $\ddot{x}=f(x, \dot{x})$. In case of the conservative systems $\ddot{x}=f(x) \equiv-\mathrm{d} W / \mathrm{d} x$, the phase portrait analysis is equivalent to the mechanical energy analysis applied in Sects. 2-6 to the present mixed convection problem. The aim of this Appendix is to show how this equivalence works, i.e., how the main characteristics of the solutions can also be recovered from the trajectories $\mathrm{d} T / \mathrm{d} y=$ functionof $(T)$ drawn in the phase plane $(T, \mathrm{~d} T / \mathrm{d} y)$ of the problem.

The whole primary information of both the mechanical energy analysis in the energy plane of Fig. 2, (particle coordinate, potential energy) $=(T, W)$ on the one hand, and of the phase portrait analysis in the phase plane (coordinate, velocity) $=(T, \mathrm{~d} T / \mathrm{d} y)$ on the other hand, is contained in the first integral (12) and the boundary conditions (6). Thus the phase paths are obtained simply either by solving the energy integral (12) with respect to $\mathrm{d} T / \mathrm{d} y$ which yields

$$
\frac{\mathrm{d} T}{\mathrm{~d} y}= \pm \sqrt{2[E-W(T)]}
$$

or by a contour plot (topographic map) of Eq. (12) for different values of the integration constant $E$. In this way, taking for example, $\left(A_{1}, A_{2}, A_{3}\right)=(1,1,100)$ and ten "representative" values of $E$, one arrives at the family of trajectories plotted in Fig. 8. Guided by the particle potential energy curve of Fig. 2, where in this case $T_{-}=-0.10512, W\left(T_{-}\right) \equiv W_{\min }=$ $\left.-0.07192), T_{+}=0.09512, W\left(T_{+}\right) \equiv W_{\max }=0.06191\right)$, the ten values of the integration constant $E$ have been selected as follows: the red phase path corresponds to the choice $E=W_{\max }=0.06191$ and represents the homoclinic orbit (curve $a$ ); the two blue paths correspond to $E=0.1$ (outer curve $b$ ) and $E=0.8$ (inner curve $b$ ), both the $E$-values being larger than $E=W_{\max }=0.06191$. The six green orbits $c$ correspond to values of $E$ in the range $W_{\min } \leq E<W_{\max }$, namely $E=0.04,0.02,0,-0.02,-0.03$ and $E=W_{\min }=-0.07192$, being enumerated according to their increasing distances from the homoclinic orbit $a$.

The closed green orbits $c$ correspond to the (bounded) periodic solutions (oscillations of the particle around the minimum of the potential well of Fig. 2). In this sense the center at $T=$ $T_{-}=-0.10512$ represents the sixth closed orbit having radius zero. The open counterparts of the green orbits $c$ correspond to unbounded solutions in the range $T>T_{+}=0.09512$. The black curve $d$ corresponds to the value $E=-0.1$ below the minimum $W_{\min }=-0.07192$ of the particle potential energy and it is also associated with an unbounded solution. In this way, the (red) homoclinic orbit $a$ separates the bounded solutions (motions) from the unbounded ones.

It is important to notice that the phase diagram of Fig. 8 characterizes the whole solution domain of Eq. (9), regardless of the boundary conditions (6). The boundary conditions $T(-1)=T(+1)=0$, however, impose two severe restrictions on the phase space trajectories corresponding to the actual solutions of the problem. In this sense (i) the mentioned trajectories must start and end on the vertical axis, $T=0$, and (ii) their "orbital period", i.e., the time spent by the representative point $\left\{T(y), T^{\prime}(y)\right\}$ on the orbit (from the initial instant $y=-1$ to the final one $y=+1$ ) must be equal to 2 . These features are illustrated in Figs. 9, 10 and 11 where the phase paths corresponding to the temperature solutions of Figs. 5, 6, and 7 have been plotted, respectively.

First of all, it is immediately seen that the two (green) inner orbits corresponding to the particle energy levels $E=-0.02$ and -0.03 do not satisfy the requirement (i) (no intersection points with the vertical axis). The third inner orbit corresponding to $E=0$ does satisfy the requirement (i) but, for the assumed values $\left(A_{1}, A_{2}, A_{3}\right)=(1,1,100)$, it 


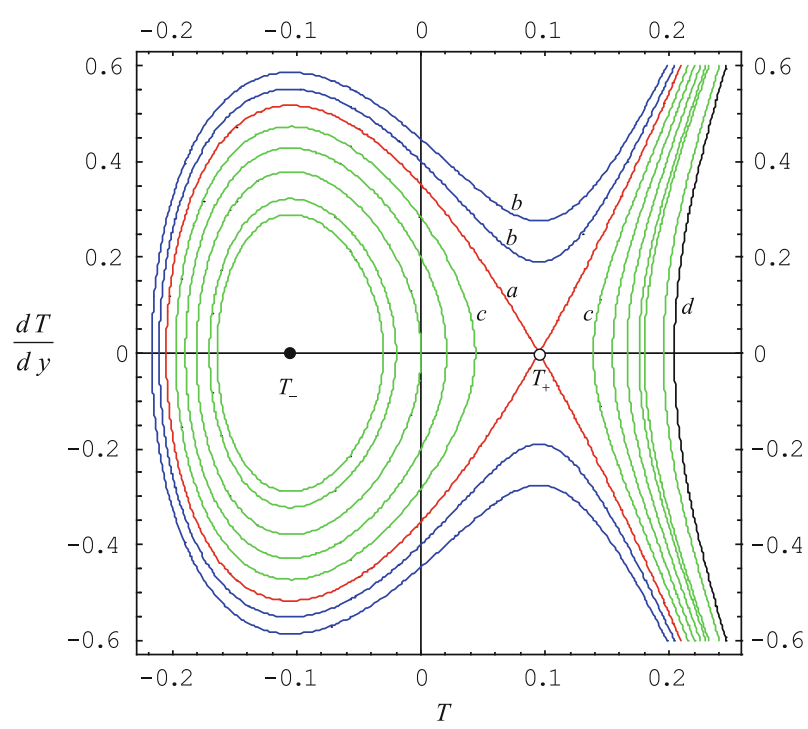

Fig. 8 Phase portrait of the solution domain of the governing differential equation (9) for the parameter values $\left(A_{1}, A_{2}, A_{3}\right)=(1,1,100)$. The phase paths correspond to ten selected values of the integration constant $E$ (see text). The "center" at $T=T_{-}=-0.10512$ (full dot) corresponds to the minimum of the particle potential energy and it is a stable equilibrium point. The "saddle-point" at $T=T_{+}=0.09512$ (open dot) with the red "homoclinic orbit" through it, corresponds to the maximum of the particle potential energy, and it is an unstable equilibrium point

violates the requirement (ii) (which can be satisfied for $E=0$ only by the adiabatic flows corresponding to the nonlinear eigenvalues $A_{3}^{(n)}$ discussed in Sect. 5; see also Fig. 11 below).

The symmetric first branch solution represented by the black curve of Fig. 5 corresponds in the phase plane of Fig. 9 to the $\operatorname{arc} A_{1} B_{1} C_{1}$ of the black orbit $a$ associated with the particle energy level $E=0.06184$ (which is quite close to the homoclinic orbit corresponding to $\left.E=W_{\max }=0.06191\right)$. The representative point $\left\{T(y), T^{\prime}(y)\right\}$ of this state moves from the initial states $A_{1}=\left\{T(-1), T^{\prime}(-1)\right\}=(0,0.351685)$ via the particle turning point $B_{1}=\left\{T(0), T^{\prime}(0)\right\} \equiv\left(T_{*,+}, 0\right)=(0.092516,0)$ toward the final state $C_{1}=$ $\left\{T(+1), T^{\prime}(+1)\right\}=(0,-0.351685)$, spending on the orbit precisely the time 2 . Thus the dashed left branch of the black orbit $a$ (in the negative range of $T$ ) has no physical significance in this case.

The asymmetric pair of temperature distributions (the red and the blue curves of Fig. 5) are represented in the phase plane by two overlapping orbits which is the pink curve $b$ of Fig. 9 and corresponds to the particle energy level $E=0.329160^{2} / 2=0.054173$. The representative point of the red temperature profile moves from the initial state $A_{2}=(0,0.329160)$ in the clockwise direction, passes the particle turning points $B_{2}$ and $B_{2}^{\prime}$, and returns to the point $A_{2}=$ $(0,0.329160)$ precisely after two time units. The representative point of the blue temperature profile moves from the initial state $C_{2}=(0,-0.329160)$ also in the clockwise direction, passes the particle turning points $B_{2}^{\prime}$ and $B_{2}$, and returns to the point $C_{2}=(0,-0.329160)$ after the same orbital period 2 .

The symmetric second branch temperature profile (the green curve of Fig. 5) is represented in the phase plane by green orbit $c$ of Fig. 9 and corresponds to the particle energy level $E=0.183253^{2} / 2=0.016791$. The representative point of this temperature solution moves in the phase plane from the initial state $A_{3}=(0,0.183253)$ also in the clockwise direction, 


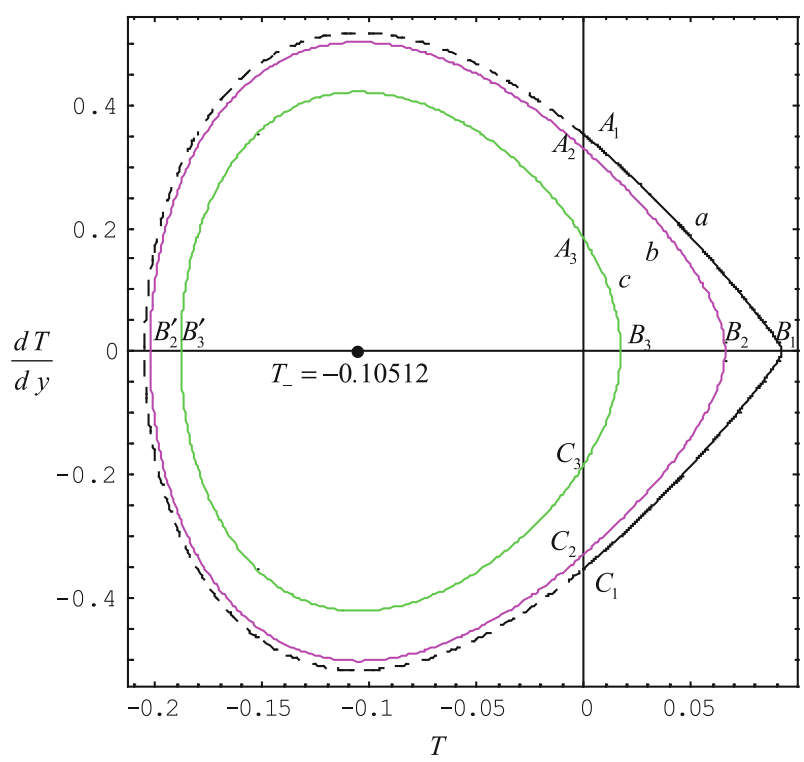

Fig. 9 Phase portrait of the temperature solutions plotted in Fig. 5

passes the particle turning points $B_{3}^{\prime}$ and $B_{3}$, arrives at the initial state $A_{3}=(0,0.183253)$ again, and continues to move toward the final state $C_{3}=(0,-0.183253)$ completing in this way in two time units the negative arc $C_{3} B_{3}^{\prime} A_{3}$ of the green orbit $c$ once and its positive arc $A_{3} B_{3} C_{3}$ twice.

In Fig. 10 the phase paths of the symmetric temperature profiles of Fig. 6 have been plotted. The corresponding particle energy levels are $E=(-0.553207)^{2} / 2=0.153019$ (red orbit), $E=(-0.200923)^{2} / 2=0.020185$ (green orbit), and $E=(-0.063143)^{2} / 2=0.001993$ (blue orbit). The representative points always move in the clockwise direction from the lower intersection points of the orbits with the vertical axis toward the respective final states which are the upper intersection points. The orbital period in all three cases is 2 . The right dashed arcs of the orbits (in the range $T>0$ ) are unphysical.

The phase portraits of the adiabatic temperature profiles plotted of Fig. 7 are shown in Fig. 11. All the orbits correspond to the same particle energy level $E=0$ and to the nonlinear eigenvalues of the buoyancy parameter $A_{3}^{(1)}=37.6952$ (black curve $a$ ), $A_{3}^{(2)}=576.5296$ (green curve $b$ ), $A_{3}^{(3)}=2892.6345$ (blue curve $c$ ), and $A_{3}^{(4)}=9113.0696$ (red curve $d$ ), respectively. The representative points always move from the initial state $\left\{T(-1), T^{\prime}(-1)\right\}=(0,0)$ in the clockwise direction until they reach the final state $\left\{T(+1), T^{\prime}(+1)\right\}=(0,0)$ after exhausting the 2 time units. Bearing in mind that the orbital periods of the four phase paths associated with the eigenvalues $A_{3}^{(n)}$ are $P_{n}=$ $2 / n, n=1,2,3,4$ (see Sect. 5), the representative point reaches the final state by going round along the black orbit $a$ once, along the green orbit $b$ twice, along the blue orbit $c$ three times, and along the red orbit $d$ four times. The number $n$ of these orbital revolutions of the phase points corresponds in terms of the mechanical analogy to the number of the complete oscillations of the particle of total particle energy $E=0$ in the respective potential wells $W_{n}(T)=-\left(A_{3}^{(n)} / 3\right) T^{3}-(1 / 2) T^{2}+T$. 


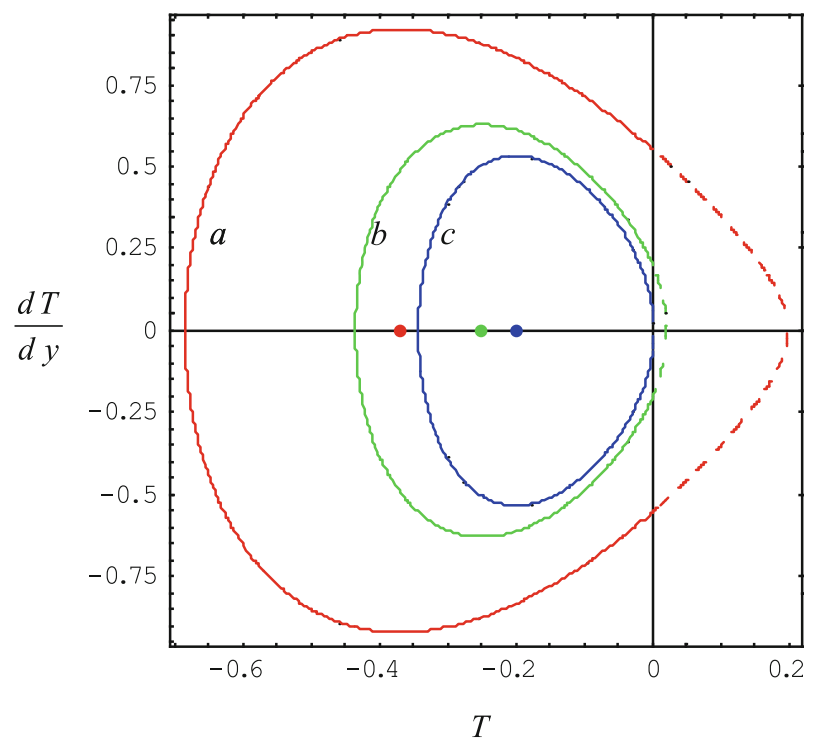

Fig. 10 Phase portrait of the temperature solutions plotted in Fig. 6 for $A_{1}=A_{2}=1$ and $A_{3}=10$ (red curve $a), A_{3}=20$ (green curve $b$ ), and $A_{3}=30$ (blue curve $c$ ). The dots indicate the corresponding centers (stable equilibrium points), $T_{-}=-0.37016,-0.25$, and -0.2 , respectively

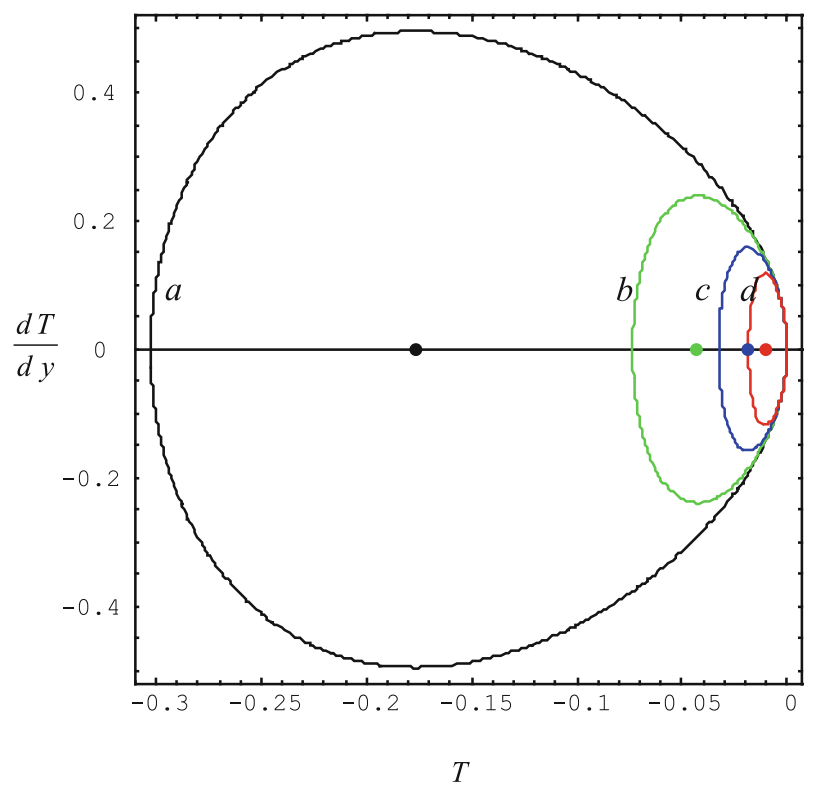

Fig. 11 Phase portraits of the adiabatic temperature profiles plotted in Fig. 7 for $A_{1}=A_{2}=1$ and the nonlinear eigenvalues of the buoyancy parameter $A_{3}^{(1)}=37.6952$ (black curve a), $A_{3}^{(2)}=576.5296$ (green curve $b), A_{3}^{(3)}=2892.6345$ (blue curve c), and $A_{3}^{(4)}=9113.0696$ (red curve $d$ ). The dots indicate the corresponding centers (stable equilibrium points): $T_{-}=-0.17668,-0.04252,-0.01877$, and -0.01053 , respectively 


\section{References}

Abramowitz, M., Stegun, I.A.: Handbook of Mathematical Functions. Dover, New York (1972)

Barletta, A., Magyari, E., Pop, I., Storesletten, L.: Unified analytical approach to the Darcy mixed convection with viscous dissipation in a vertical channel. Int. J. Therm. Sci. 47, 408-416 (2008)

Barletta, A., Nield, D.A.: Combined forced and free convective flow in a vertical porous channel: the effects of viscous dissipation and pressure work. Transp. Porous Media 79, 319-334 (2009a)

Barletta, A., Nield, D.A.: Response to comment on "Combined forced and free convective flow in a vertical porous channel: The effects of viscous dissipation and pressure work" by A. Barletta and D. A. Nield. Transp. Porous Media, 79, 319-334 (2009a). Transp. Porous Media 80, 397-398 (2009b)

Kuznetsov, A.V.: Analytical investigation of heat transfer in Couette flow through a porous medium utilizing the Brinkman-Forchheimer-extended Darcy model. Acta Mech 129, 213-224 (1998)

Magyari, E.: Comment on "Combined Forced and Free Convective Flow in a Vertical Porous Channel: The Effects of Viscous Dissipation and Pressure Work" by A. Barletta and D. A. Nield, Transp. Porous Media, Transp. Porous Media, 79, 319-334 (2009a). Transp. Porous Media 80, 389-395 (2009a)

Magyari, E.: Further comments on "Combined Forced and Free Convective Flow in a Vertical Porous Channel: The Effects of Viscous Dissipation and Pressure Work". Transp. Porous Media 80, 399-400 (2009b)

Nield, D.A., Junqueira, S.L.M., Lage, J.L.: Forced convection in a fluid-saturated porous-medium channel with isothermal or isoflux boundaries. J. Fluid Mech. 322, 201-214 (1996)

Umavathy, J.C., Kumar, J.P., Chamkha, A.J., Pop, I.: Mixed convection in a vertical porous channel. Transp. Porous Media 61, 315-335 (2005)

Umavathy, J.C., Kumar, J.P., Chamkha, A.J., Pop, I.: Erratum: mixed convection in a vertical porous channel. Transp. Porous Media 75, 129-132 (2008) 